\title{
Active Control of Structures with Uncertain Coupled Subsystems and Actuator Dynamics
}

\author{
Ningsu Luo, Rodolfo Villamizar, José Rodellar and Josep Vehí
}

\begin{abstract}
This paper deals with the problem of stabilizing a class of structures subject to an uncertain excitation due to the temporary coupling of the main system with another uncertain dynamical subsystem. A Lyapunov function based control scheme is proposed to attenuate the structural vibration. In the control design, the actuator dynamics is taken into account. The control scheme is implemented by using only feedback information of the main system. The effectiveness of the control scheme is shown for a bridge platform with crossing vehicle.
\end{abstract}

\section{INTRODUCTION}

Vibrations in dynamical flexible structures, as those encountered in some civil engineering structures, are often caused by environmental (seismic or wind) excitations and human made (traffic or heavy machinery) excitations. One way for attenuating the structural vibrations is to use the active control systems so that the safety of the structure and comfortability of the human beings are improved ${ }^{[1 !}$. Robust control methods have been used to account for uncertainties in the structural models and the lack of knowledge of the excitations $[2]-[6]$. This paper considers a class of structures whose excitation comes through the uncertain coupling with another dynamical system during a certain time. One prototype of this class of systems is illustrated by considering a bridge platform with an unknown moving vehicle as a coupled exciting subsystem. A Lyapunov based control scheme is proposed to reduce the vibration of bridge induced by the crossing vehicle. In the control design, only the feedback information from the controlled structure (bridge) is used. Numerical simulation is done to show the effectiveness of the proposed active control scheme for an elastically suspended bridge when a truck crosses it.

\section{PROBLEM FORMULATION}

Consider the problem of controlling an elastically suspended bridge with active elastic mounts on the left-hand and right-hand sides and with crossing vehicles as shown in Figure 1. The main variables to be measured are the vertical deviation $z$ of the center of mass of the bridge and the inclination $\theta$ with respect to the horizon of the bridge platform. Vibration of the bridge is produced when a truck crosses the bridge with velocity $v(t)$ within a time interval $\left[t_{0}, t_{f}\right]$. Without the loss of generality, $t_{0}$ is set to

This work has been funded by the Commission of Science and Technology of Spain (CICYT) under the Project DPI2002-04018

Ningsu Luo, Rodolfo Villamizar and Josep Vehi are with Institute of Informatics and Applications, University of Girona, 17071 Girona, Spain <ningsu, rodolfov, vehi@eia.udg.es

José Rodellar is with the School of Civil Engineering, Technical University of Catalonia, 08034, Barcelona, Spain jose.rodellar@upc.es zero and $t_{f}$ denotes the final time of interaction between the structure and the truck. The truck is modelled by a mass $m$ with an elastic suspension of damping $c$ and stiffness $k$. Additional variables $\xi, \eta$ and $\zeta$ are chosen according to Figure 1 . The mass of the platform is given by $M$, and the moment of inertia with respect to $\dot{C}$ by the parameter $J$. The active control is implemented by two actuators located between the ground and the bridge at the left and the right ends respectively. The actuators $A_{1}$ and $A_{2}$ supply vertical control forces $M u_{1}$ and $M u_{2}$ which complement the resistant passive forces $F_{1}$ and $F_{2}$ given by the elastic supports. $u_{1}$ and $u_{2}$ are the control variables that relate the forces supplied by the active actuators $A_{1}$ and $A_{2}$. The objective is to attenuate the vibration of the bridge induced by the crossing vehicle by using active forces $M u_{1}$ and $M u_{2}$.

\section{A. Equation of motion of the truck:}

When the truck is not in the bridge (for $t<0$ and $t>t_{f}$ ), the equation of motion of the truck is $m \ddot{\eta}=k \eta_{0}-m g$, where $\eta_{0}$ is the position of relaxed suspension. When $t \in$ $\left[0, t_{f}\right]$, the truck is crossing the bridge. Assume that the declination angle $\Theta$ is small, then the dynamic motion of the truck is described by the following equation

$$
\left\{\begin{array}{l}
m \ddot{\eta}=F-m g \\
F:=k\left[\dot{\eta}_{0}-(\eta+\zeta)\right]-c(\dot{\eta}+\dot{\zeta}) \\
\zeta:=z+(\xi-a) \Theta
\end{array}\right.
$$

\section{B. Equations of motion of the bridge:}

For $t<0$ the bridge is in a steady state. For $t \in\left[0, t_{f}\right]$, the dynamic behavior of the bridge is described by the following equations of motion:

$$
\left\{\begin{array}{l}
M \ddot{z}=M g+F-F_{1}-F_{2}-u_{1}-u_{2} \\
J \ddot{\Theta}=(\xi-a) F+a F_{1}-b F_{2}+a u_{1}-b u_{2} \\
F:=k\left[\eta_{0}-(\eta+\zeta)\right]-c(\dot{\eta}+\dot{\zeta}) \\
F_{1}=k_{1}\left(-z_{1,0}+z-a \Theta\right)+c_{1}(\dot{z}-a \dot{\Theta}) \\
F_{2}=k_{2}\left(-z_{2,0}+z+b \Theta\right)+c_{2}(\dot{z}+b \dot{\Theta})
\end{array}\right.
$$

where $z_{1,0}$ and $z_{2,0}$ represent the vertical positions of relaxed left-hand and right-hand suspension, respectively.

We consider the bridge as the main system and the truck as the attached uncertain subsystem. The space state variables are split into the measurable ones, $\mathbf{x}:=(z, \Theta, \dot{z}, \dot{\Theta})^{T}$, and the unmeasurable ones $\mathbf{y}:=(\eta, \dot{\eta})^{T} . \mathbf{u}:=\left(u_{1}, u_{2}\right)^{T}$ are control forces. The uncertain coupling between the bridge and the truck is due to the scalar force $F$. When 
the truck has left the bridge for $t>t_{f}$, the two systems are obviously decoupled with $F=0$ and then the equations of motion of the bridge are

$$
\left\{\begin{array}{l}
M \ddot{z}=M g-F_{1}-F_{2}-u_{1}-u_{2}, \\
J \ddot{\theta}=a F_{1}-b F_{2}+a u_{1}-b u_{2} .
\end{array}\right.
$$

In the above models, consider that the structural parameters of the bridge $\left(M, J, c_{1}, c_{2}, k_{1}, k_{2}\right)$ are known, while the parameters related to the truck $\left(m, c, k, \eta_{0}, \xi, \dot{\xi}\right)$ are assumed to be uncertain but bounded; i.e.,

$$
\begin{aligned}
& \frac{k}{m}=\omega_{0}+\Delta \omega, \quad \text { with }|\Delta \omega| \leq \bar{\omega}, \\
& \frac{c}{m}=\sigma_{0}+\Delta \sigma, \quad \text { with }|\Delta \sigma| \leq \bar{\sigma}, \\
& \frac{k}{M}=\Omega, \quad \text { with } \Omega \leq \bar{\Omega}, \\
& \frac{c}{M}=\Upsilon, \quad \text { with } \Upsilon \leq \bar{\Upsilon}, \\
&\left|\eta_{0}\right| \leq \bar{\eta}_{0}, \quad|\dot{\xi}(t)| \leq \bar{\xi}
\end{aligned}
$$

where $\omega_{0}$ and $\sigma_{0}$ are known nominal values and $\bar{\omega}, \bar{\sigma}, \bar{\Omega}, \bar{\Upsilon}$, $\dot{\xi}$ and $\bar{\eta}_{0}$ are known bounds. Finally the equations of motion (1) and (2) can be rewritten into the following form:

$$
\left\{\begin{aligned}
\dot{\mathbf{x}} & =\mathbf{A}_{\mathbf{c}} \mathbf{x}+\mathbf{B u}+\mathbf{g}(\mathbf{x}, \mathbf{y}, t), \\
\dot{\mathbf{y}} & =\mathbf{A}_{\mathbf{r}} \mathbf{y}+\mathbf{f}(\mathbf{x}, \mathbf{y}, t)
\end{aligned}\right.
$$

where the parameters of the matrices $\mathbf{A}_{\mathbf{c}}, \mathbf{B}$ and $\mathbf{A}_{\mathbf{r}}$ are known. The functions $g$ and $f$ include the uncertain coupling effects.

$$
\begin{aligned}
& \mathbf{A}_{\mathbf{c}}=\left(\begin{array}{cc}
0 & 0 \\
0 & 0 \\
-\frac{k_{1}+k_{2}}{M} & \frac{a k_{1}-b k_{2}}{M} \\
\frac{a k_{1}-b k_{2}}{J} & -\frac{a^{2} k_{1}+b^{2} k_{2}}{J}
\end{array}\right. \\
& \left.\begin{array}{cc}
1 & 0 \\
0 & 1 \\
-\frac{c_{1}+c_{2}}{M} & \frac{a c_{1}-b c_{2}}{M} \\
\frac{a c_{1}-b c_{2}}{J} & -\frac{a^{2} c_{1}+b^{2} c_{2}}{J}
\end{array}\right) \\
& \mathbf{B}=\left(\begin{array}{cc}
0 & 0 \\
0 & 0 \\
-\frac{1}{M} & -\frac{1}{M} \\
\frac{a}{J} & -\frac{b}{J}
\end{array}\right), \text { and } \mathbf{g}=\left(\begin{array}{c}
0 \\
0 \\
g_{3} \\
g_{4}
\end{array}\right)
\end{aligned}
$$

Here for $t \in\left[0, t_{f}\right]$ :

$$
\begin{aligned}
g_{3}(\mathbf{x}, \mathbf{y}, \dot{t}):= & -\frac{k}{M} z-\frac{1}{M}[k(\xi-a)+c \dot{\xi}] \Theta-\frac{c}{M} \dot{z} \\
& -\frac{c}{M}(\xi-a) \dot{\Theta}-\frac{k}{M} \eta-\frac{c}{M} \dot{\eta}+\frac{k}{M} \eta_{0} \\
& +\frac{k_{1}}{M} z_{1,0}+\frac{k_{2}}{M} z_{2,0}+g
\end{aligned}
$$

$$
\begin{aligned}
g_{4}(\mathbf{x}, \mathbf{y}, t):= & -\frac{k}{J}(\xi-a) z-\frac{1}{J}\left[k(\xi-a)^{2}+c \dot{\xi}(\xi-a)\right] \\
& \Theta-\frac{c}{J}(\xi-a) \dot{z}-\frac{c}{J}(\xi-a)^{2} \dot{\Theta}-\frac{k}{J}(\xi-a) \\
& \eta-\frac{c}{J}(\xi-a) \dot{\eta}+\frac{k}{J}(\xi-a) \eta_{0}-\frac{a k_{1}}{J} z_{1,0} \\
& +\frac{b k_{2}}{J} z_{2,0}
\end{aligned}
$$

while, for $t>t_{f}$,

$$
\begin{gathered}
g_{3}:=\frac{k_{1}}{M} z_{1,0}+\frac{k_{2}}{M} z_{2,0}+g \\
g_{4}:=-\frac{a k_{1}}{J} z_{1,0}+\frac{b k_{2}}{J} z_{2,0} \\
\mathbf{A}_{\mathbf{r}}=\left(\begin{array}{cc}
0 & 1 \\
-\omega_{0} & -\sigma_{0}
\end{array}\right) \\
\mathbf{f}(\mathbf{x}, \mathbf{y}, t)=\left(\begin{array}{c}
0 \\
\mathbf{f}_{2}
\end{array}\right)
\end{gathered}
$$

For $t \in\left[0, t_{f}\right]$,

$$
\begin{aligned}
\mathbf{f}_{2}= & -\frac{k}{m} z-\frac{1}{m}[k(\xi-a)+c \dot{\xi}] \theta-\frac{c}{m} \dot{z}-\frac{c}{m} \\
& (\xi-a) \dot{\theta}-\Delta \omega \eta-\Delta \sigma \dot{\eta}+\frac{k}{m} \eta_{0}-g
\end{aligned}
$$

and for $t>t_{f}$,

$$
\mathrm{f}_{2}=-\Delta \omega \eta-\Delta \sigma \dot{\eta}+\frac{k}{m} \eta_{0}-g
$$

Denote $\mathbf{e}=\left(e_{1}, e_{2}\right)^{T}$

$$
\begin{aligned}
e_{i}(\mathbf{x}, \mathbf{y}, t)= & e_{i, 1}(t) z+e_{i, 2}(t) \Theta+e_{i, 3}(t) \dot{z}+e_{i, 4}(t) \dot{\Theta} \\
& +e_{i, 5}(t) \eta+e_{i, 6}(t) \dot{\eta}+e_{i, 7}(t)
\end{aligned}
$$

Now, it can be verified that $\mathbf{A}_{\mathbf{c}}$ and $\mathbf{A}_{\mathbf{r}}$ are stable matrices and the function $\mathbf{e}(\mathbf{x}, \mathbf{y}, \cdot)$ is continuous for all $t$ except a set $\left\{0, t_{f}\right\}$ and there exist known non-negative scalars $\alpha_{i}^{c}$, $\alpha_{i}^{r}, \delta_{i}$, such that, for all $\mathbf{x}, \mathbf{y}$ and $t$, one has

$$
\mathbf{g}=\left[\mathbf{B}_{1}, \mathbf{B}_{2}\right]\left[\mathbf{e}_{1}, \mathbf{e}_{2}\right]^{T}
$$

where

$$
\mathbf{B}_{1}=\left(\begin{array}{c}
0 \\
0 \\
-\frac{1}{M} \\
\frac{a}{J}
\end{array}\right), \quad \mathbf{B}_{2}=\left(\begin{array}{c}
0 \\
0 \\
-\frac{1}{M} \\
-\frac{b}{J}
\end{array}\right)
$$

and

$$
\left\|\mathbf{e}_{i}(\mathbf{x}, \mathbf{y}, t)\right\| \leq \alpha_{i}^{c}\|\mathbf{x}\|+\alpha_{i}^{r}\|\mathbf{y}\|+\delta_{i}
$$

with

$$
\begin{aligned}
\alpha_{i}^{\mathrm{c}} & =\sqrt{\alpha_{1}^{2}+\alpha_{2}^{2}+\alpha_{3}^{2}+\alpha_{4}^{2}}, \\
\alpha_{i}^{r} & =\sqrt{\alpha_{5}^{2}+\alpha_{6}^{2}}, \\
\delta_{i} & =\alpha_{7} .
\end{aligned}
$$


where

$$
\begin{aligned}
& \alpha_{1}=\bar{\Omega} \\
& \alpha_{2}= \begin{cases}\frac{1}{(a+b)}\left(\bar{\Omega} a^{2}+(a \bar{\Omega}+\bar{\Upsilon} \overline{\dot{\xi}}) a+a \bar{\Upsilon} \bar{\xi}\right), & \text { if } a \geq b \\
\frac{1}{(a+b)}\left(\bar{\Omega} b^{2}+(b \bar{\Omega}+\bar{\Upsilon} \overline{\dot{\xi}}) b+b \bar{\Upsilon} \overline{\dot{\xi}}\right), & \text { if } a<b\end{cases} \\
& \alpha_{3}=\vec{\Upsilon} \\
& \alpha_{4}= \begin{cases}\frac{2 a^{2}}{(a+b)} \bar{\Upsilon}, & \text { if } a \geq b \\
\frac{2 b^{2}}{(a+b)} \bar{\Upsilon}, & \text { if } a<b\end{cases} \\
& \alpha_{5}=\bar{\Omega} \\
& \alpha_{6}=\bar{\Upsilon} \\
& \alpha_{7}=\max \left\{\frac{1}{(a+b)}\left[\bar{\Omega}(a+b) \vec{\eta}_{0}+\frac{(a+b) k_{1} z_{1,0}+g}{M}\right]\right. \text {, } \\
& \left.\frac{1}{(a+b)}\left[\bar{\Omega}(a+b) \bar{\eta}_{0}+\frac{(a+b) k_{2} z_{2,0}+a g}{M}\right]\right\}
\end{aligned}
$$

Indeed, solving the linear system $\mathbf{g}=\mathbf{B e}$, it is easy to get that $\mathbf{e}=\left(e_{1}, e_{2}\right)^{T}$, where

$$
e_{1}=\frac{-b M g_{c, 3}+J g_{c, 4}}{(a+b) M} ; \quad e_{2}=-\frac{a M g_{c, 3}+J g_{c, 4}}{(a+b) M}
$$

\section{CONTROLLER DESIGN}

The objective of active control is to attenuate the vibration of the bridge induced by a crossing truck through the uncertain coupling between the dynamics of the bridge and the truck. The controller design will be based on

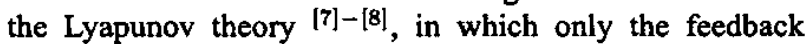
information of the bridge (not the truck) is used.

In order to design the controller, define a Lyapunov function candidate:

$$
V(x)=\frac{1}{2} x^{T}(t) P x(t)
$$

where $\boldsymbol{P}=\left(p_{i j}\right) \in R^{4 \times 4}$ is the positive definite solution of the Lyapunov equation

$$
\boldsymbol{P A}+\boldsymbol{A}^{T} \boldsymbol{P}+\boldsymbol{Q}=\mathbf{0}
$$

for a given symmetric positive definite matrix $\boldsymbol{Q}=\left(q_{i j}\right) \in$ $R^{4 \times 4}$. By using equations (31)-(32), the derivative of $V(x, t)$ is obtained

$$
\begin{aligned}
& \qquad \begin{array}{ll}
\dot{V}(\boldsymbol{x}, t) & =\boldsymbol{x}^{T} \boldsymbol{P} \boldsymbol{B}_{1} u_{1}+\boldsymbol{x}^{T} \boldsymbol{P} \boldsymbol{B}_{2} u_{2}+x^{T} \boldsymbol{P} \boldsymbol{B}_{1} e_{\mathbf{1}} \\
& +\boldsymbol{x}^{T} \boldsymbol{P} \boldsymbol{B}_{2} e_{2}-\frac{1}{2} \boldsymbol{x}^{T} \boldsymbol{Q} \boldsymbol{x} \\
& \leq H(\boldsymbol{x}, \boldsymbol{u})+H(\boldsymbol{y})
\end{array} \\
& \qquad \\
& \qquad \begin{array}{l}
H(\boldsymbol{y})=:\left(\alpha_{1}^{r}\left\|\boldsymbol{x}^{T} \boldsymbol{P} \boldsymbol{B}_{1}\right\|+\alpha_{2}^{r}\left\|\boldsymbol{x}^{T} \boldsymbol{P} \boldsymbol{B}_{2}\right\|\right) \cdot\|\boldsymbol{y}\|
\end{array}
\end{aligned}
$$

where

and

$$
H(\boldsymbol{x}, \boldsymbol{u})=: H_{1}\left(\boldsymbol{x}, \boldsymbol{u}_{1}\right)+H_{2}\left(\boldsymbol{x}, \boldsymbol{u}_{2}\right)-\frac{1}{2} \boldsymbol{x}^{T} \boldsymbol{Q} \boldsymbol{x}
$$

where

$$
\begin{aligned}
H_{i}\left(\boldsymbol{x}, u_{i}\right)= & \delta_{i}\left\|\boldsymbol{x}^{T} \boldsymbol{P} \boldsymbol{B}_{i}\right\|+\alpha_{i}^{c}\left\|\boldsymbol{x}^{T} \boldsymbol{P} \boldsymbol{B}_{i}\right\| \cdot\|\boldsymbol{x}\| \\
& +\boldsymbol{x}^{T} \boldsymbol{P} \boldsymbol{B}_{i} u_{i}
\end{aligned}
$$

Since the state variable $y(t)$ of the coupled uncertain subsystem (the truck) is usually not measurable, the objective of control is to minimize the $\dot{V}(x)$ by making the $H(\boldsymbol{x}, \boldsymbol{u})<0$. If we denote $\boldsymbol{u}_{i}^{d}(t)$ as the "desired" control force (without taking into account the actuator dynamics), then the following "desired" control law will be used :

$$
\boldsymbol{u}_{i}^{d}=-\boldsymbol{k}_{i}^{p} \boldsymbol{x}-\left(\delta_{i}+\alpha_{i}^{c}\|\boldsymbol{x}\|\right) \operatorname{sgn}\left(\boldsymbol{x}^{T} \boldsymbol{P} \boldsymbol{B}_{i}\right)
$$

where

$$
\boldsymbol{k}_{i}^{p}=\frac{1}{4} \boldsymbol{B}_{i}^{T} \boldsymbol{P}
$$

It is easy to verify that $H(\boldsymbol{x}, \boldsymbol{u})<0$ is accomplished. In practice, the continuous approximation is used for the control law (35) to attenuate the high-frequency chattering

$$
\operatorname{sgn}(\cdot) \Longrightarrow \frac{(\cdot)}{|(\cdot)|+\gamma}
$$

where $\gamma$ is a positive small constant. Thus, the corresponding continuous "desired" control law is

$$
\boldsymbol{u}_{i}^{d}=-k_{i}^{p} x-\left(\delta_{i}+\alpha_{i}^{c}\|x\|\right) \frac{x^{T} P B_{i}}{\left|x^{T} \boldsymbol{P} B_{i}\right|+\gamma_{i}}
$$

Now, assume that active actuators, the $A_{1}$ and $A_{2}$ one, are used for the implementation of the control action generated by the "desired" controllers (35) or (38). The dynamic behavior of the active actuators is described by the following equation ${ }^{[9]}$ :

$$
v_{i}(t)=\tau_{i} \dot{u}_{i}(t)+u_{i}(t)+k_{i}^{f} \dot{z}(t), \quad i=1,2
$$

where $u_{i}(t)$ is the average output actuator force, $v_{i}(t)$ the voltage signal applied to the actuator, $\tau$ is the actuator time delay and $k_{i}^{f}$ is a constant which relates the friction force produced in the actuator with the velocity of the piston. For simplicity, in the subsequent sections $u_{i}(t)$ and $v_{i}(t)$ will be called the actuator control force and actuator control command, respectively. By taking into account the actuator dynamics, a control command law $v(t)$ is designed such that the "real" actuator control force $u(t)$ tracks asymptotically 
the "desired" actuator control force $u_{d}(t)$ before obtained, which.

Denote $\tilde{\boldsymbol{u}}(t)$ as the tracking error between the "real" control action $u(t)$ and the "desired" control action $u^{d}(t)$; i.e.,

$$
\tilde{u}(t)=u(t)-u^{d}(t)
$$

Suppose that the actuator parameters $\tau$ and $k^{f}$ are known positive constants and the velocity $\dot{z}$ measurable. Then the following command control law is proposed:

$$
v_{i}(t)=\tau_{i} \dot{u}_{i}^{d}(t)+u_{i}^{d}(t)+k_{i}^{f} \dot{z}(t), \quad i=1,2
$$

Apply the real control force $u(t)=\left[u_{1}(t), u_{2}(t)\right]^{T}(39)$ to the bridge platform and define a new Lyapunov function candidate $V(\boldsymbol{x}, \tilde{\boldsymbol{u}})$

$$
V(\boldsymbol{x}, \tilde{\boldsymbol{u}})=V_{1}(\boldsymbol{x})+V_{2}(\tilde{\boldsymbol{u}})
$$

with

$$
V_{1}(\boldsymbol{x})=\frac{1}{2} x^{T} \boldsymbol{P} \boldsymbol{x} \quad V_{2}(\tilde{\boldsymbol{u}})=\frac{1}{2} \tilde{\boldsymbol{u}}^{T} \boldsymbol{\tau} \tilde{\boldsymbol{u}}
$$

where $\tau=\operatorname{diag}\left(\tau_{1}, \tau_{2}\right)$

¿From the eqn.(40) we obtain:

$$
\boldsymbol{u}(t)=\boldsymbol{u}_{d}(t)+\tilde{\boldsymbol{u}}(t)
$$

Then, the derivative of $V(x, \tilde{\boldsymbol{u}})$ is obtained as follows by using eqns. (41, 35), (39) and (40):

$$
\dot{V}(\boldsymbol{x}, \tilde{\boldsymbol{u}}) \leq H(\boldsymbol{x}, \boldsymbol{u}, \tilde{\boldsymbol{u}})+H(\boldsymbol{y})
$$

where

$$
\begin{aligned}
H(\boldsymbol{x}, \boldsymbol{u}, \tilde{\boldsymbol{u}})=: \quad & H(\boldsymbol{x}, \boldsymbol{u})-\tilde{\boldsymbol{u}}^{T} \tilde{\boldsymbol{u}}-\frac{1}{4} \boldsymbol{x}^{T} \boldsymbol{P} \boldsymbol{B} \boldsymbol{B}^{T} \boldsymbol{P} \boldsymbol{x} \\
& +\boldsymbol{x}^{T} \boldsymbol{P B} \tilde{\boldsymbol{u}} \\
= & H(\boldsymbol{x}, \boldsymbol{u})-\left(\tilde{\boldsymbol{u}}-\frac{1}{2} \boldsymbol{x}^{T} \boldsymbol{P B}\right)^{T}(\tilde{\boldsymbol{u}} \\
& \left.-\frac{1}{2} x^{T} \boldsymbol{P B}\right) \leq H(\boldsymbol{x}, \boldsymbol{u})<0
\end{aligned}
$$

Therefore, the "real" control force $u(t)$ (taking into account the actuator dynamics) can minimize the derivative of Lyapunov function $\dot{V}(\boldsymbol{x}, \overline{\boldsymbol{u}})$ by making $H(\boldsymbol{x}, \boldsymbol{u}, \tilde{\boldsymbol{u}})<0$, which is similar to the case when a "desired" control force $\boldsymbol{u}_{d}(t)$ (without taking into account the actuator dynamics) is applied to the bridge platform.

\section{NUMERICAL SIMULATION RESULTS}

In the numerical simulation, an actively suspended bridge platform prototype is considered as the main system and the excitation is induced by a truck when it crosses the bridge [10]. The following parameters are used for the controller design and numerical simulation:

A. Nominal parameters and bounds for uncertainties:

$\bar{\eta}_{0}=1[\mathrm{~m}], \omega_{0}=40[\mathrm{~N} /(\mathrm{m} \mathrm{kg})], \vec{\omega}=20[\mathrm{~N} /(\mathrm{m} \mathrm{kg})]$, $\sigma_{0}=1[\mathrm{Ns} /(\mathrm{m} \mathrm{Kg})], \bar{\sigma}=5[\mathrm{Ns} /(\mathrm{m} \mathrm{Kg})], \bar{\Omega}=5[\mathrm{~N} /(\mathrm{m}$ $\mathrm{kg})], \bar{\Upsilon}=0.5[\mathrm{Ns} /(\mathrm{m} \mathrm{Kg})], \overline{\dot{\xi}}=8.33[\mathrm{~m} / \mathrm{s}](\dot{\xi}=30[\mathrm{~km} / \mathrm{h}])$, $k_{0}=4 \cdot 10^{5}[\mathrm{~N} / \mathrm{m}], c_{0}=10^{4}[\mathrm{Ns} / \mathrm{m}]$.

\section{B. Bridge:}

$M=10^{5} \mathrm{Kg}, J=2 \cdot 10^{7} \mathrm{Kg} \mathrm{m}^{2}, a=b=25 \mathrm{~m}, k_{i}=4 \cdot 10^{6}$ $\mathrm{N} / \mathrm{m}$ and $c_{i}=4 \cdot 10^{4} \mathrm{~N} \mathrm{~s} / \mathrm{m}$ for each $i=1,2 . z_{1,0}=z_{2,0}=$ $-0.125 \mathrm{~m}$, which correspond to the equilibrium position for the platform without truck and no control.

C. Truck:

The parameters of the truck, which are unknown for the controller design, are the following: $m=10^{4} \mathrm{Kg}, \dot{\xi}=8.33$ $\mathrm{m} / \mathrm{s}(\dot{\xi}=30 \mathrm{Km} / \mathrm{h}), k=4 \cdot 10^{5} \mathrm{~N} / \mathrm{m}, c=10^{4} \mathrm{~N} \mathrm{~s} / \mathrm{m}$, $\eta_{0}=0.75 \mathrm{~m}$.

D. Active actuators [9]:

$k_{l}^{f}=k_{2}^{f} \doteq 15 \mathrm{~kg} / \mathrm{s}$ and $\tau_{1}=\tau_{2}=0.18 \mathrm{~s}$.

With the above parameters, we obtain:

$\alpha=\left[\begin{array}{lllllll}5 & 129.165 & 0.5 & 12.5 & 5 & 0.5 & 500.0025\end{array}\right]^{T}$
$\alpha_{1}^{c}=\alpha_{2}^{c}=129.8657, \delta_{1}^{c}=\delta_{2}^{c}=5 \times 10^{-5}, \gamma_{1}=\gamma_{2}=0.01$
$k_{1}^{p}=\left[\begin{array}{llll}-0.0016 & 0.0001 & -0.1582 & 0.0063\end{array}\right]$
$k_{2}^{p}=\left[\begin{array}{llll}-0.0016 & -0.0001 & -0.1582 & -0.0063\end{array}\right]$

The platform is excited by the crossing of the truck for time $t \in[0,6]$ seconds, and after $t=6$ seconds no excitation is evolved between the platform and the truck. The time history of structural vibration of the bridge platform for the uncontrolled case (dash line) and the controlled case (solid line) are shown in Figures 2 and 3. Concretely, Figure 2 shows the main effect of the control, which is to add damping to the bridge platform. Without control, the platform has very low damping, thus exhibiting a highly oscillatory response. The damping coefficients of the two end supports are $c_{1}=c_{2}=4 \times 10^{4} \mathrm{~N} \mathrm{~s} / \mathrm{m}$, which corresponds to a damping factor of $4.5 \%$ approximately. The control modifies this behavior, forcing a practically overdamped response. It is seen how the vertical deflection $z$ of the center of mass of the platform evolves slowly but smoothly towards its equilibrium position with the truck. After $t=6$ seconds the excitation disappears and the platform deflection evolves to recover the initial equilibrium position. Figure 4 shows that the inclination $\theta$ of the bridge has not been significantly improved because the linear control. Figure 5 displays the vertical displacement of the truck which has not been deteriored and figures 6 and 7 display the control signals $u_{1}$ and $u_{2}$, which are feasible for practical actuators.

\section{CONCLUSIONS}

An active Lyapunov based control scheme has been proposed in this paper to attenuate the vibrations of a main system excited by an temporarily coupled uncertain subsystem. Only the feedback information of the main system has been used in the control design, without measuring the response of the coupled uncertain subsystem. It has been shown that the active controller also works well when the actuator dynamics is taken into account. The results of 
numerical simulation have illustrated the effectiveness of the proposed control scheme for an active controlled suspended bridge platform with crossing vehicles.

\section{REFERENCES}

[1] Housner, et al., Structural control: past, present and future, Journal of Engineering Mechanics, 897-971, 1997.

[2] J.M. Kelly, G. Leitmann and A. Soldatos, Robust control of base isolated structures under earthquake excitation, J. Optim. Theory Appl., 53, 159-180, 1987.

[3] J. Rodellar, G. Leitmann E.P. Ryan, Output feedback control of uncertain coupled systems, International Journal of Control, Vol. 58, pp. 445-457, 1993.

[4] A.H. Barbat, J. Rodellar, E.P. Ryan and N. Molinares, Active control of non-linear base-isolated buildings, Journal of Engineering Mechanics ASCE, Vol. 121, 676-684, 1995.

[5] N. Luo, J. Rodellar and M. De la Sen, Composite robust active control of seismically excited structures with actuator dynamics, Earthquake Engineering and Structural Dynamics, Vol. 27, pp. 301$311,1998$.

[6] E. Reithmeier and G. Leitmann, Structural vibration control, Journal of the Franklin Institute, Vol. 338, pp. 203-223, 2000.

[7] N. Luo, J. Rodellar and M. De la Sen, Sliding mode control of flexible structures, Advances in Structural Control, Editorial: CIMNE, Barcelona, J. Rodellar, A. Barbat, F. Casciati (eds.), 1999.

[8] V.I. Utkin, Sliding Modes in Control and Optimization, SpringerVerlag, Berlin, Heidelberg, 1992.

[9] J. Ghaboussi and A. Joghataia, Active control of structures using neural networks, Journal of Engineering Mechanics ASCE, Vol. 121, 4, 555-567, 1995.

[10] J. Rodellar, V. Mañosa, E. Reithmeier and G. Erhet, Vibration control of structures with uncertainties due to coupled subsystems, in Proceedings of the European Control Conference, FEUP ediçoes, Porto 2001, 78-83, 2001

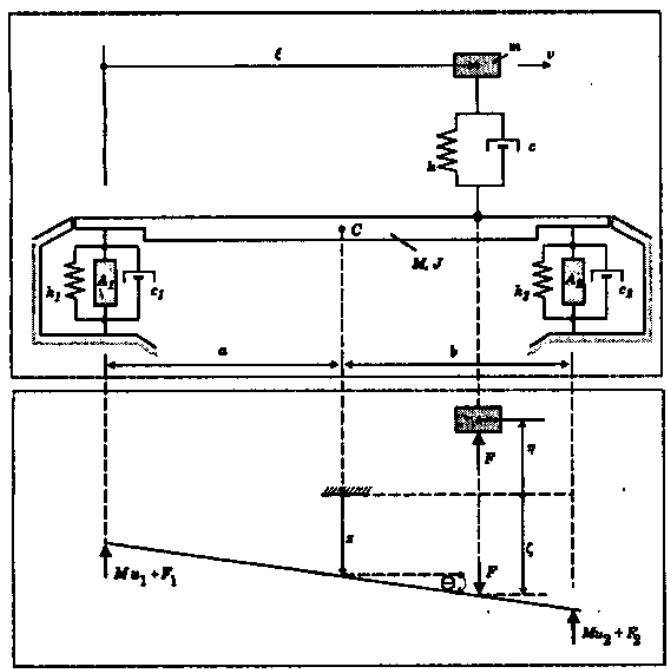

Fig. 1. Actively controlled bridge platform with crossing vehicle

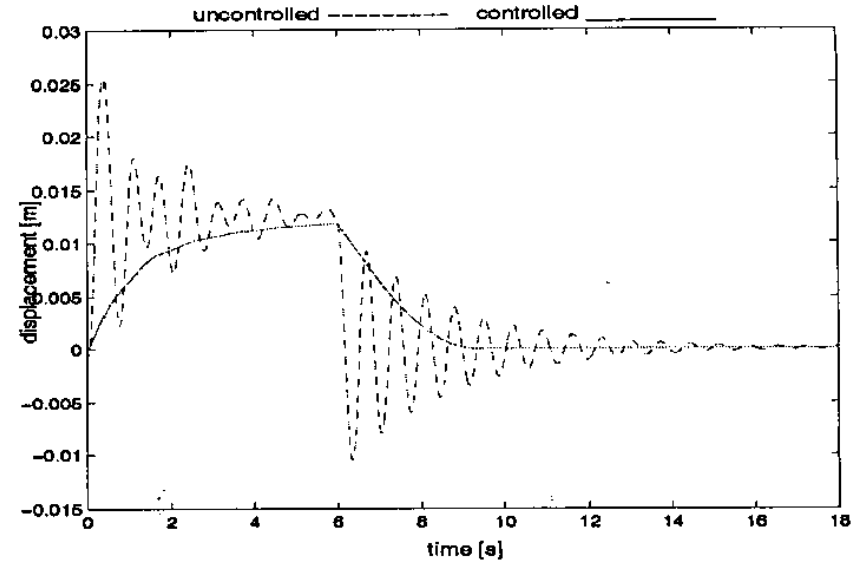

Fig. 2. Vertical displacement of the bridge

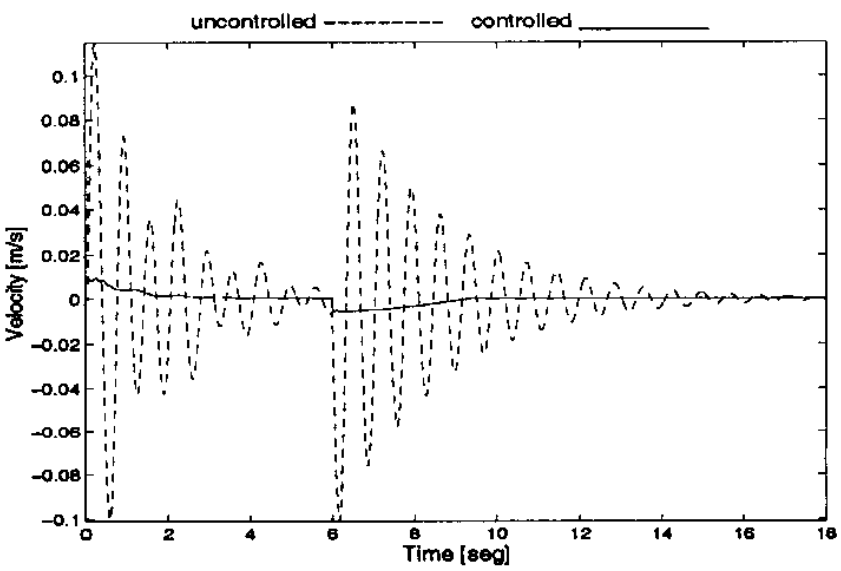

Fig. 3. Vertical velocity of the bridge

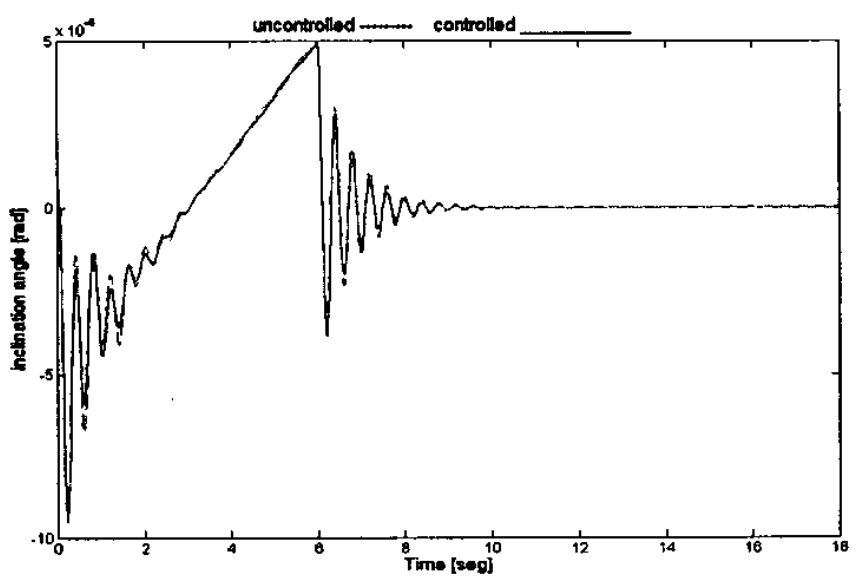

Fig. 4. Inclination angle of the bridge 


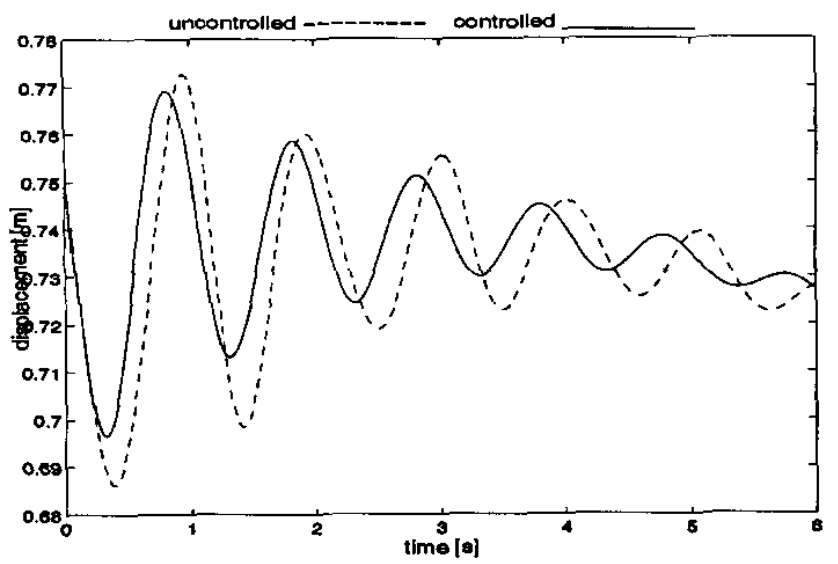

Fig. 5. Vertical displacement of the truck

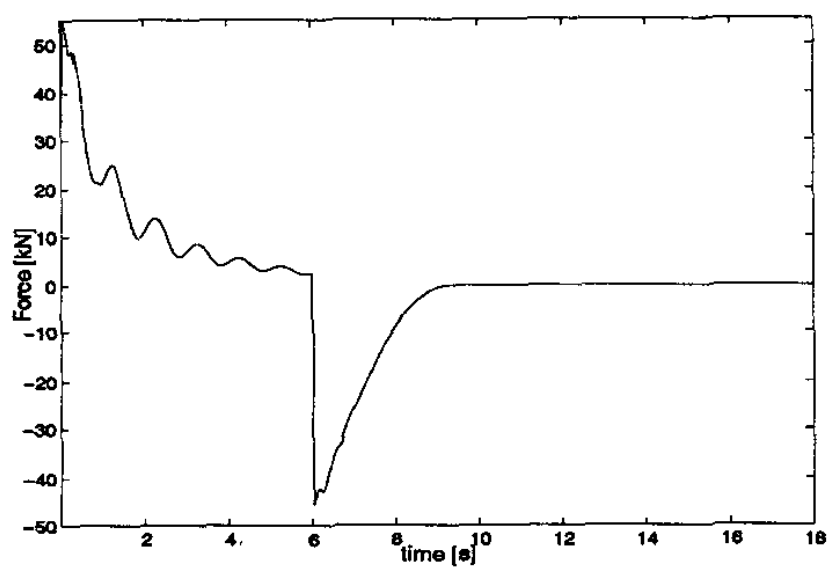

Fig. 6. Control force of the actuator Al

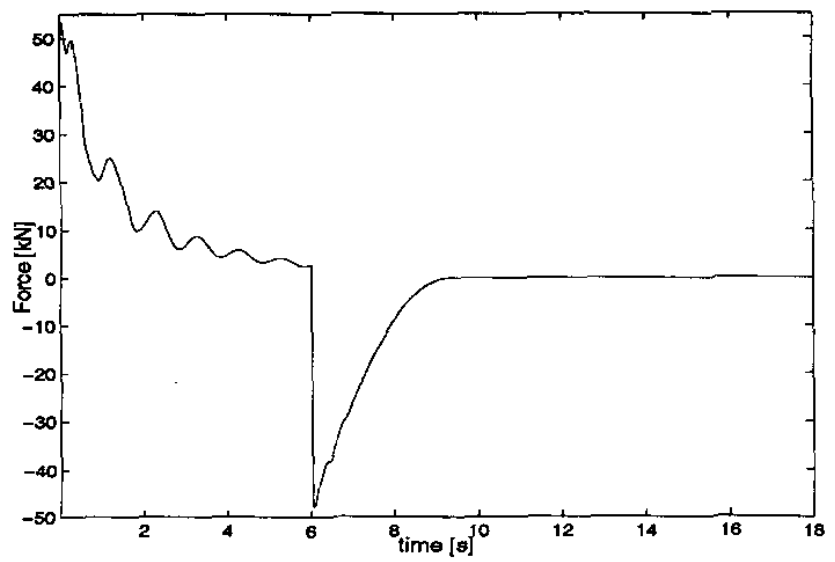

Fig. 7. Control force of the actuator A2 